\title{
Prevalence and predictive factors for the detection of carcinoma in cavity margin performed at the time of breast lumpectomy
}

\author{
Iulia Tengher-Barna ${ }^{1}$, Delphine Hequet ${ }^{2}$, Jeanne Reboul-Marty ${ }^{3}$, Annonciade Frassati- \\ Biaggi $^{1}$, Nathalie Seince ${ }^{2}$, Anabela Rodrigues-Faure ${ }^{2}$, Michèle Uzan ${ }^{2}$ and Marianne Ziol ${ }^{1}$ \\ ${ }^{1}$ Service d'Anatomie et de Cytologie Pathologiques, CHU Jean Verdier AP-HP, Bondy, France; ${ }^{2}$ Service de \\ Gynécologie-obstétrique, CHU Jean Verdier AP-HP, Bondy, France and ${ }^{3}$ Service de Santé Publique, CHU Jean \\ Verdier AP-HP, Bondy, France
}

\begin{abstract}
Margin resection status is a major risk factor for the development of local recurrence in breast conservation therapy for carcinoma. Tumor bed excision sent as separate orientated cavity margins represents a tool to verify the completeness of the carcinoma resection. We aimed to (1) determine the prevalence of positive cavity margin and its influence on subsequent surgical treatment and (2) identify potential predictive factors for positive cavity margins. From 2003 to 2006,107 (57 years; 30-88) consecutive patients who underwent a lumpectomy for carcinoma with four orientated cavity margins for carcinoma were selected. Preoperative clinical, radiological and histological data, perioperative macroscopic characteristics and definitive histological analysis results were recorded. Lumpectomy or cavity margins were considered as positive when the distance from carcinoma to the margin was less than or equal to $3 \mathrm{~mm}$. Histological examination of cavity margins showed carcinoma in 38 patients (35\%), therefore modifying subsequent surgical therapy in 33 cases. Examination of the cavity margins led (1) to avoiding surgical re-excision in 20 cases (lumpectomy margins were positive and the cavity margins negative), (2) to performing a mastectomy or a re-excision in 13 cases (carcinoma was detected in the cavity margins although the lumpectomy margins were negative or tumor size was superior to $3 \mathrm{~cm}$ ). Between preoperative and perioperative parameters, US scan and macroscopic size of the tumor were predictive factors for positive cavity margins whereas characteristics of the carcinoma determined on biopsy samples and macroscopic status of the lumpectomy margins were not. Our study confirms that the systematic practice of cavity margin resection avoids surgical re-excision and reduces the likelihood of underestimating the extent of the tumor.
\end{abstract}

Modern Pathology (2009) 22, 299-305; doi:10.1038/modpathol.2008.186; published online 7 November 2008

Keywords: breast carcinoma; breast conserving surgery; lumpectomy; margin; histopathology

Breast conservation treatment constitutes the standard procedure in early stage breast cancer. ${ }^{1}$ Close or involved margins are a major risk factor for the development of local recurrence ${ }^{2,3}$ and systemic disease. ${ }^{4,5}$ Thus, the surgeon will attempt to obtain a widely clear rim of benign tissue surrounding the carcinoma and routine histopathological analysis of lumpectomy specimen partly consists of careful assessment of all margins. Specimens are oriented with sutures by the surgeon and inked by the pathologist. All margins are examined and the

Correspondence: Professor M Ziol, MD, PhD, Service d'Anatomie et de Cytologie pathologique, Hôpital Jean Verdier AP-HP, Avenue du 14 Juillet, 93143 Bondy, France.

E-mail: marianne.ziol@jvr.aphp.fr

Received 15 July 2008; revised 23 September 2008; accepted 29 September 2008; published online 7 November 2008 microscopic distance between infiltrating and/or in situ carcinoma and inked margins are determined. There is no consensus as to what constitutes a positive or negative margin, ${ }^{6,7}$ but we considered like others a margin superior or equal to $3 \mathrm{~mm}$ as negative. $^{8}$ If the margin is less than $3 \mathrm{~mm}$, a re-excision is proposed to achieve a completely clear margin, as radiotherapy alone does not compensate for incomplete surgery. ${ }^{9}$

However, the friability of the lumpectomy specimen, the lack of definite shape of the adipose tissue, its ability to be distorted (compression of the specimen for radiographs), ${ }^{10}$ render the assessment of margins uncertain with the risk of false positivity. Therefore, some surgeons take additional pieces of breast tissue from each side of the lumpectomy cavity. These separate cavity margins are oriented by suture and the new margin is inked. Some beneficial effects 
of this procedure that supersede the lumpectomy have been reported in retrospective studies ${ }^{7,11,12}$ as the cavity margin procedure significantly decreased the need for re-excision. Although this procedure seems to reduce the need for re-excision in our institution, we also experienced several unexpected occurrences of cavity margin positive for carcinoma or carcinoma in situ whereas lumpectomy margins were negative. These findings on the cavity margin sample led to re-excision or mastectomy when the total size of the carcinoma exceeded $3 \mathrm{~cm}$ or presented as a multifocal carcinoma. Patients' age, tumor size in histological report, extensive intraductal component, high histological grade and lymph node metastasis have been reported as factors associated with positive margin, ${ }^{7,11-13}$ but the predictive value of preoperative factors such as radiologic size or peroperative factors such as macroscopic assessment of margin have so far not been investigated.

We aimed (1) to evaluate the frequency of the detection of carcinoma in cavity margin and its influence on patient's subsequent surgical therapy therapeutic management and (2) to identify potential preoperative or peroperative factors associated with positive cavity margin.

\section{Patients and methods}

\section{Patient Selection}

From January 2003 to December 2006, all consecutive patients who underwent lumpectomy for breast carcinoma at our institution were selected. In all cases, a preoperative diagnosis of carcinoma, either invasive, intraductal or both, had been established on micro- or macrobiopsy with determination of the histological type, histological grade according to Ellston and Ellis, hormonal receptors and HER2 status, and proliferation index. The lumpectomies were performed by only two surgeons (NS, ARF) specialized in breast surgery following the same procedure for unifocal tumors of less than $3 \mathrm{~cm}$. In addition to an oriented lumpectomy with two sutures, four additional cavity margins were taken from the wall of the residual cavity: superior, inferior, medial and lateral. The protocol of our surgical team consisted of an excision extending from the subcutis to the pectoral fascia. Therefore, neither anterior nor posterior cavity margins were taken.

All patients' charts were reviewed and the following information was collected: age at diagnosis, personal or familial history of large bowel, ovarian or breast cancer, hormonal status, size of the tumor determined on US scan and presence of microcalcification.

\section{Pathologic Examination}

From 2003 to 2006, pathologic examination was standardized with forms for macroscopy and histological analysis. The orientated lumpectomy specimens were promptly delivered to the pathologist, measured in three dimensions and opened to determine intraoperatively the greatest dimension of the tumor. The distance from the tumor to the margin (superior, inferior, medial, lateral) was macroscopically determined on the fresh specimen and recorded. No frozen section's examination was performed. Lumpectomy margins were then inked in two different colors and the specimen was fixed in formalin. Multiple sections were performed to sample tumor and evaluate lateral, medial, superior and inferior margins so that all lumpectomy specimen was transferred into tissue blocks. Four separate cavity margin (lateral, medial, superior and inferior) were orientated by a suture placed on the cavity side of the specimen and further measured in three dimensions and inked. Cavity margin were fully sectioned into 3- to 4-mm-thick slices and transferred into blocks. Each lumpectomy and cavity margin block was examined on one hematoxylineosin-saffron stained slide. Lumpectomy margins were considered as positive when the distance from the carcinoma (either invasive or in situ) to the medial, lateral, superior or posterior was less than or equal to $3 \mathrm{~mm}$. Lumpectomy margin positivity was considered as focal when the distance from the carcinoma was less than $3 \mathrm{~mm}$ on only one block tissue section and as extensive when observed on two or more than two blocks. Cavity margins were positive when either carcinoma in situ or invasive carcinoma was detected. The margins of the cavity margin were considered positive when carcinoma was identified at less than or equal to $3 \mathrm{~mm}$ from any of the inked borders of the four cavity margins. When any of these cavity margins were positive or the total size of the tumor (determined histologically) exceeded $3 \mathrm{~cm}$ or if it was bifocal, patients were recommended a re-excision or a mastectomy. The maximal size of both invasive carcinoma and invasive and intraductal carcinoma was determined on histological sections. Invasive ductal carcinoma with extensive intraductal component was defined as tumor with a more than $25 \%$ intraductal component. When the highest dimension of the tumor (invasive and intraductal) was higher than the highest dimension of the invasive carcinoma, it was defined as carcinoma with intraductal component encompassing invasive component. Bi- or multifocality was defined by the presence of two or more foci of invasive carcinoma separated by normal tissue. ${ }^{14}$ In cases of subsequent re-excisions, the specimen was completely embedded. In cases of subsequent mastectomies, specimens were sliced at $0.5-1 \mathrm{~cm}$ intervals and all suspicious areas were sampled for histological examination. Regardless of suspicious areas, 10 tissue specimen were systematically taken from the bed of the initial lumpectomy. In addition, at least one sample of each quadrant of the breast was systematically examined along with the nipple and the retroareolar area. 
Histological assessment of cavity margin changed the subsequent surgical management in three situations: (1) when the margins of the cavity margins were free of carcinoma whereas lumpectomy margins were positive, surgical re-excision was avoided; (2) when cavity margin disclosed a multifocal carcinoma or when the conglomerated tumor size on both the lumpectomy and cavity margins outlined measured more than $3 \mathrm{~cm}$, a mastectomy was recommended; (3) when the margins of the cavity margin were positive whereas lumpectomy margin were negative, a re-excision or mastectomy was recommended.

\section{Statistical Analysis}

Quantitative variables were described using the median and the range. Nominal variables were described using the frequency and the percentage.

For univariate analysis, we tested the association of the carcinoma in cavity margins with pre-, periand postoperative factors with the $\chi^{2}$-tests for nominal variables and with the Mann-Whitney test for quantitative variables.

For multivariate analysis, significant variables in the univariate analysis were used in logistic regression analyses to identify multivariate predictors of the detection of the carcinoma in cavity margins. Stepwise procedures were used to select those variables associated with the carcinoma in cavity margins. A significance level of 0.10 is required to allow a variable to enter into or to stay in the model. All the statistical significances were tested by the likelihood ratio test. Data sets were prepared and analyzed with SAS 9.1. All $P$-values reported are two-sided with a significant level of 0.05.

\section{Results}

\section{Patient's Preoperative Characteristics}

One hundred and seven patients underwent lumpectomy for carcinoma from January 2003 to December 2006. They were aged 30-88 years (median 57) and US scan size of the tumor, when measurable (in 99 of the 107 cases), ranged from 4 to $30 \mathrm{~mm}(\mathrm{mean}=15.7 \mathrm{~mm})$. Eight patients had microcalcifications with no tumor detectable by US scan. Preoperative characteristics of patients and histological assessment of carcinoma on biopsy samples are detailed in Table 1.

\section{Lumpectomy and Cavity Margin Status}

Lumpectomy margin were positive in 47 patients; margin involvement was focal in 20 cases and extensive in 27 cases. Cavity margin were positive (containing either invasive carcinoma in 16 cases or in situ carcinoma alone in 22 cases) in 38 patients $(35 \%)$. A single cavity margin was positive in 20
Table 1 Preoperative patient/tumor characteristics

$\begin{array}{lc}\text { Age at diagnosis: median (range) (years) } & 57(30-88) \\ \text { Menopause } & 70(65 \%) \\ \text { Menopause with HRT } & 24(22 \%) \\ \text { Personal or familial history of cancer (\%) } & 31(29 \%) \\ \text { Tumor size (US scan) measured before surgery } & \\ \quad \text { Median (range) (mm) } & 15.7(4-30) \\ & \\ \text { Histological type (determined on biopsies) } & 80(75 \%) \\ \text { Invasive ductal carcinoma } & 12(11 \%) \\ \text { Invasive lobular carcinoma } & 15(14 \%) \\ \text { Ductal carcinoma in situ } & \\ & \\ \text { Tumor grade } & 38(41 \%) \\ \text { I } & 41(45 \%) \\ \text { II } & 13(14 \%) \\ \text { III } & 73(79 \%) \\ \text { Estrogen receptor }+ & 58(63 \%) \\ \text { Progesterone receptor }+ & 20(22 \%) \\ \text { C-erbB2 }+ & \end{array}$

HRT, hormone replacement therapy.

patients whereas 18 patients had 2-4 positive cavity margins. Among the latter, $27(71 \%)$ patients had positive lumpectomy margin (6 with focal involvement, 21 with extensive involvement) but 9 (29\%) had negative lumpectomy margin. Localization of lumpectomy margin involvement was consistent with cavity margin positivity in most cases (19 out of 27). As depicted in Figure 1, examination of the cavity margins, by itself, modified subsequent surgical therapy in 33 patients. (1) When lumpectomy margin were positive and cavity margin free of carcinoma, surgical re-excision was avoided $(n=20$ cases); (2) when cavity margin were positive despite negative lumpectomy margin $(n=9)$, surgical re-excision or mastectomy was offered and (3) when cavity margin examination disclosed multifocality or a carcinoma of more than $30 \mathrm{~mm}(n=4)$, surgical re-excision or mastectomy was offered. More precisely, among the nine patients with positive cavity margin and negative lumpectomy margin, five had several foci of ductal carcinoma in situ detected in cavity margin and four had invasive ductal carcinoma. Regarding perioperative macroscopic assessment, lumpectomy margin were macroscopically suspected to be positive in 34 cases but truly positive at histological examination in 24 cases. Positive predictive value of macroscopic perioperative assessment was $70 \%$. Lumpectomy margin appeared macroscopically free of tumor in 73 cases but carcinoma was detected at histological examination in 23 cases. Negative predictive value of macroscopic assessment was $68 \%$. As shown in Figure 2, 32 of the 107 patients underwent subsequent mastectomy or re-excision and residual carcinoma was detected in 15 patients (47\%). More precisely, two out of the nine patients with negative lumpectomy margin and positive cavity margin had residual carcinoma and two out of three patients who had a tumor size above $3 \mathrm{~cm}$ revealed by cavity margin analysis also had residual carcinoma. 
Average macroscopic volume of the lumpectomy was $76 \mathrm{~cm}^{3}$ (range: 10-217) and average volume of the sum of the four cavity margins was $40 \mathrm{~cm}^{3}$ (range 21-120).

\section{Factors Associated with the Detection of Carcinoma in Cavity Margins}

Preoperative characteristics associated with the detection of carcinoma in cavity margin (Table 2)

\begin{tabular}{|l|l|l|l|l|l|}
\hline & $\begin{array}{l}\text { Lumpectomy } \\
\text { margin }\end{array}$ & Cavity margin & $\begin{array}{l}\text { Margin of the } \\
\text { canty margin }\end{array}$ & $\begin{array}{l}\text { Number of } \\
\text { patients }\end{array}$ & $\begin{array}{l}\text { Cavity margin } \\
\text { assessment } \\
\text { modfied therapy }\end{array}$ \\
\hline- & - & - & 49 & no \\
\hline+ & + & - & 20 & yes" \\
\hline
\end{tabular}

Figure 1 Influence of margin status on surgical management. The central circle represents the lumpectomy and the four crescents represent the four cavity margins. Black stars represent the carcinoma either invasive and or intraductal. Positive margins (distance of the carcinoma to the margin $\leq 3 \mathrm{~mm}$ ) are identified on the figure when the tumor touches the limits of the lumpectomy or of the cavity margins. LM, lumpectomy margin; CM, cavity margin and MCM, margin of the cavity margin. * Assessment of cavity margin avoided further surgical therapy and **led to reexcision or mastectomy. were the US scan determined size of the tumor and the non-menopausal status. Others parameters such as a history of cancer, histological type and grade, hormonal receptor and HER2 status were not associated with positive cavity margins. It was the macroscopically assessed size of the tumor and not the lumpectomy margins status that was predictive of the presence of carcinoma in cavity margins. Among parameters retrieved from histological analysis (Table 2), the size of either invasive or intraductal carcinoma, the detection of lymph node metastasis and the detection of carcinoma in lumpectomy margins were significantly associated with positive cavity margin in the univariate study. Multivariate analysis taking into account all preand perioperative characteristics shows that US scan size and non-menopausal status were independently related to positive cavity margins. Taking into account all parameters, only lymph node metastasis and the size of carcinoma (including both invasive and intraductal components) were independently related to positive cavity margins. Odd ratio with confidence intervals are given in Table 3 . When patients were segregated according to US scan size ( $\leq$ or $>10 \mathrm{~mm}$ ) after excluding lobular carcinoma, negative cavity margins could be predicted in $91 \%$ of cases ( 3 false negative cases out of 34 cases with US scan size below $10 \mathrm{~mm}$ ). Using this US scan size threshold, sensitivity and specificity for positive cavity margin were 89 and $52 \%$, respectively.

\section{Discussion}

Close or involved margins are a major risk factor for the development of local recurrence ${ }^{4}$ in both invasive and in situ ductal carcinoma. To minimize the risk of local recurrence, several approaches including cavity margin resection have been used.

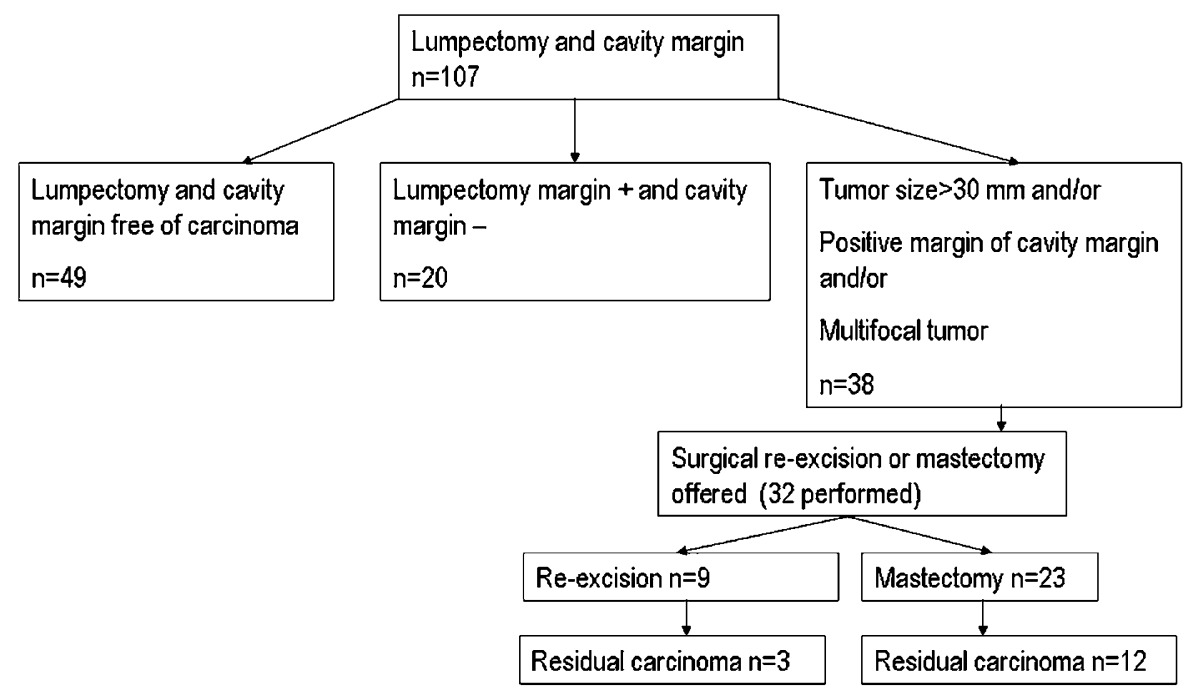

Figure 2 Flow chart summarising surgical treatment, margin status and residual disease in 107 patients with breast conservation therapy. 
Table 2 Pre-, peri- and postoperative factors associated with the detection of carcinoma in cavity margins

\begin{tabular}{|c|c|c|c|}
\hline Number of patients & $\begin{array}{l}\text { Cavity margins negative } \\
\qquad(\mathrm{n}=69)\end{array}$ & $\begin{array}{l}\text { Cavity margins positive } \\
\qquad(\mathrm{n}=38)\end{array}$ & $\mathrm{P}$ \\
\hline \multicolumn{4}{|l|}{ Preoperative characteristics } \\
\hline Age at surgery & $60(37-88)$ & $57(30-86)$ & 0.2 \\
\hline Menopause & $51(74 \%)$ & $19(50 \%)$ & 0.01 \\
\hline Personal history of cancer & $4(6 \%)$ & $2(5 \%)$ & 0.9 \\
\hline Familial history of cancer & $16(23 \%)$ & $10(28 \%)$ & 0.8 \\
\hline Microcalcifications & $3(4 \%)$ & $5(13 \%)$ & 0.13 \\
\hline US scan size of the tumor & 14.3 & 18.9 & 0.005 \\
\hline \multicolumn{4}{|l|}{ Histological characteristics on biopsy samples } \\
\hline Histological type of carcinoma & & & 0.5 \\
\hline Invasive lobular & $6(9 \%)$ & $6(16 \%)$ & \\
\hline Invasive ductal & $54(78 \%)$ & $26(68 \%)$ & \\
\hline In situ ductal & $9(13 \%)$ & $6(16 \%)$ & \\
\hline Histological grade ${ }^{\mathrm{a}}$ & & & 0.5 \\
\hline I & $28(46 \%)$ & $10(31 \%)$ & \\
\hline II & $24(40 \%)$ & $17(53 \%)$ & \\
\hline III & $8(14 \%)$ & $5(16 \%)$ & \\
\hline Estrogens receptor $+^{\mathrm{a}}$ & $46(76 \%)$ & $27(84 \%)$ & 0.5 \\
\hline Progesterone receptor $t^{\mathrm{a}}$ & $36(60 \%)$ & $22(68 \%)$ & 0.5 \\
\hline c-erbB2 $+^{\mathrm{a}}$ & $13(21 \%)$ & $7(22 \%)$ & 0.8 \\
\hline Proliferation index $($ Ki67 $)>30 \%{ }^{a}$ & $15(25 \%)$ & $9(28 \%)$ & 0.9 \\
\hline \multicolumn{4}{|l|}{ Perioperative characteristics } \\
\hline Size of the tumour (gross finding) & $16 \pm 9$ & $20.7 \pm 8$ & 0.02 \\
\hline Positive margin (gross finding) & $19(27 \%)$ & $14(37 \%)$ & 0.4 \\
\hline \multicolumn{4}{|l|}{ Postoperative characteristics } \\
\hline \multicolumn{4}{|l|}{ Pathological size } \\
\hline Of invasive carcinoma & $15(1-35)$ & $25(4-75)$ & 0.0005 \\
\hline Of in situ and invasive carcinoma & $18(4-35)$ & $34(11-75)$ & $<0.0001$ \\
\hline Histological type & & & 0.09 \\
\hline Invasive ductal carcinoma & $49(72 \%)$ & $18(47 \%)$ & \\
\hline $\begin{array}{l}\text { Invasive ductal carcinoma with extensive intraductal } \\
\text { component }\end{array}$ & $7(10 \%)$ & $9(24 \%)$ & \\
\hline Invasive lobular carcinoma & $6(8 \%)$ & $5(13 \%)$ & \\
\hline In situ ductal carcinoma & $7(10 \%)$ & $6(16 \%)$ & \\
\hline Intraductal component encompassing invasive component & $20(34 \%)$ & $18(56 \%)$ & 0.07 \\
\hline Peritumoral lymphovascular invasion & $10(16 \%)$ & $10(31 \%)$ & 0.1 \\
\hline Axillary lymph node involvement & $11(16 \%)$ & $16(42 \%)$ & 0.006 \\
\hline Lumpectomy margins & & & $<0.0001$ \\
\hline Distance from carcinoma to margins $>3 \mathrm{~mm}$ & 49 & 11 & \\
\hline Distance from carcinoma to margins $\leq 3 \mathrm{~mm}$ focally & 14 & 6 & \\
\hline Distance from carcinoma to margins $\leq 3 \mathrm{~mm}$ extensive & 6 & 2 & \\
\hline
\end{tabular}

${ }^{\mathrm{a}}$ Determined for invasive carcinoma; $n=92$.

Table 3 Multivariate analysis: characteristics associated with the presence of carcinoma in cavity margin

\begin{tabular}{llll}
\hline & Odds-ratio & 95\% CI & P-value \\
\hline Pre- and perioperative characteristics & & \\
$\quad$ US scan size & 0.95 & $0.90-0.99$ & 0.04 \\
$\quad$ Menopause & 0.38 & $0.15-0.94$ & 0.04 \\
& & & \\
All characteristics & & & \\
$\quad$ Histological size of carcinoma & 0.90 & $0.85-0.96$ & 0.006 \\
$\quad$ Lymph node metastasis & 4.27 & $1.30-13.84$ & 0.01 \\
\hline
\end{tabular}

In this study we investigated retrospectively the influence of cavity margin excision on further surgical management by comparing the therapeutic options that would have been taken with lumpectomy alone to that taken with the results of cavity margin examination. Overall, cavity margin examination changed the surgical therapy recommendations for 33 patients $(30 \%)$. On the one hand, it led to surgical re-excision being avoided in 20 patients $(19 \%)$ as lumpectomy margin was positive and cavity margin negative and on the other, it led to 
recommending a mastectomy or a re-excision in 12 cases $(11 \%)$ because carcinoma was detected in cavity margin whereas lumpectomy margin were negative or because cavity margin examination led to a carcinoma of more than $3 \mathrm{~cm}$ being disclosed. As for avoiding returning to the operating theater, our results can be compared with three previous published studies, ${ }^{7,11,15}$ which reported 61 out of $126(48 \%), 60$ out of $301(20 \%)$ and 16 out of 45 $(35 \%)$ negative cavity margin whereas lumpectomy margins were positive. Although our results are consistent with the two later studies, they did not reach half of the cases as described in the Cao et $a l^{11}$ study. This could be related to the surgical procedure as superficial and deep cavity margin were taken, assuming that resection did not extended to the skin and the pectoralis fascia. In our study, positive superficial and deep margin were not considered prerequisites to recommending further surgery and therefore were not taken into account when considering lumpectomy margin as positive. This probably explains the high prevalence of positive lumpectomy margin $(81 \%)$ in the Cao et $a l^{11}$ study.

Noteworthy is our finding that 11 of the 107 patients $(10 \%)$ had negative lumpectomy margins but positive cavity margin. This frequency is higher than reported in previous studies of cavity margins as it varied from $2,{ }^{15} 4^{7}$ to $8 \% .^{11}$ However, these results are conflicting as $9 \%$ of the tumor bed biopsies were positive in one study Rubin et al ${ }^{16}$ and $21 \%$ of the reexcision specimens also contained residual carcinoma, ${ }^{17}$ whereas the initial lumpectomy margins were free of carcinoma. Whether these false negative cases in our study were due to sampling errors (carcinoma present elsewhere in the block but not represented on the tissue section observed) or to a discontinuous localization of carcinoma cannot be determined by this study. However, the high prevalence of in situ ductal carcinoma or of invasive carcinoma with extensive intraductal components in this group of patients (9 out of 11) favors the hypothesis of skip lesions. Additional sampling represented by examination of cavity margin, especially in ductal carcinoma in situ and invasive carcinoma with extensive intraductal component, allows the risk of missing residual carcinoma to be reduced and may influence the local recurrence rate. Moreover, cavity margin examination disclosed a tumor of more than $3 \mathrm{~cm}$ in four cases and therefore to mastectomy being offered whereas lumpectomy margin examination alone would have led to re-excision being performed. Some drawbacks are linked to the systematic resection of cavity margins such as the larger volume of excision with potential adverse cosmetic effect and the time-consuming character of processing and examining four additional cavity margin (8-20 additional tissue blocks). However, avoiding a second surgical resection in $20 \%$ of cases or preventing understaging of the tumor in an addi- tional $10 \%$ of cases appears to confirm the efficacy of this method in obtaining free margins. To our knowledge, no prospective or retrospective study compared the efficacy of the cavity margin procedure vs lumpectomy alone in term of local recurrence. However, whether obtaining free margin in one surgical procedure or two has an effect on prognosis was addressed and suggested a trend toward a higher local recurrence if more than one procedure was required. ${ }^{18}$

Regarding predictive factors for carcinoma in cavity margins, we found that US scan tumor size and non-menopausal status were the only independent predictive factors for positive cavity margin. None of the characteristics determined on biopsy samples, neither macroscopic assessment of margin resection were predictive factors. Among 33 cases with macroscopic positive margin, 14 (37\%) had positive cavity margin at microscopic examination. This is in line with the Balch et al ${ }^{19}$ study reporting that gross examination of the resection specimen does not reflect margin status in at least $25 \%$ of women. We provide here further evidence that perioperative macroscopic assessment has a limited value for margin assessment. Several studies have addressed the question of patient or tumor characteristics associated with positive lumpectomy margins. By multivariate analysis, positive lumpectomy margins were associated with large tumor size $^{20,21}$ and the presence of an extensive intraductal component. ${ }^{21}$ Similarly, positive cavity margins were reported to be associated with large tumor size and tumor type (intraductal carcinoma, invasive lobular carcinoma) by multivariate analysis. ${ }^{7}$ Our results are concordant, as the distance of the initial lumpectomy margin to the carcinoma, the larger size of the tumor and metastatic axillary lymph node appeared as factors associated with positive cavity margins in univariate analysis whereas the latter two were only associated by multivariate analysis. Finally, we found that a US scan size below or equal to $10 \mathrm{~mm}$, after excluding lobular carcinoma in situ, could predict negative cavity margins in $91 \%$ of cases (31 out of 34 ). This could suggest that no cavity margin resection is needed in ductal carcinoma with a US scan size below $10 \mathrm{~mm}$. However, in 10 out of these 34 cases, lumpectomy margins were positive despite negative cavity margin and would have indicated reoperation.

In conclusion, our study confirms that the systematic practice of cavity margin resection avoids surgical re-excision in a significant number of cases and reduces the likelihood of underestimating the extent of the tumor. Excepting tumor size, neither preoperative nor perioperative factors can reliably predict the status of cavity margin.

\section{Disclosure/conflict of interest}

The authors declare no conflict of interests. 


\section{References}

1 Blichert-Toft M, Rose C, Anderson JA. Danish randomized trial comparing breast conservation therapy with mastectomy: six years of life-table analysis. Monogr Natl Cancer Inst 1992;11:19-25.

2 Kurtz JM. Factors influencing the risk of local recurrence in the breast. Eur J Cancer 1992;28:660-666.

3 Macmillan RD, Purushotham AD, George WD. Local recurrence after breast-conserving surgery for breast cancer. Br J Surg 1996;83:149-155.

4 Singletary SE. Surgical margins in patients with earlystage breast cancer treated with breast conservation therapy. Am J Surg 2002;184:383-393.

5 Fisher B, Anderson S, Fisher ER, et al. Significance of ipsilateral breast tumour recurrence after lumpectomy. Lancet 1991;338:327-331.

6 Taghian A, Mohiuddin M, Jagsi R, et al. Current perceptions regarding surgical margin status after breast-conserving therapy: results of a survey. Ann Surg 2005;241:629-639.

7 Keskek M, Kothari M, Ardehali B, et al. Factors predisposing to cavity margin positivity following conservation surgery for breast cancer. Eur J Surg Oncol 2004;30:1058-1064.

8 Pittinger TP, Maronian NC, Poulter CA, et al. Importance of margin status in outcome of breast-conserving surgery for carcinoma. Surgery 1994;116:605-608.

9 Leong C, Boyages J, Jayasinghe UW, et al. Effect of margins on ipsilateral breast tumor recurrence after breast conservation therapy for lymph node-negative breast carcinoma. Cancer 2004;100:1823-1832.

10 Dooley WC, Parker J. Understanding the mechanisms creating false positive lumpectomy margins. Am J Surg 2005;190:606-608.

11 Cao D, Lin C, Woo SH, et al. Separate cavity margin sampling at the time of initial breast lumpectomy significantly reduces the need for reexcisions. Am J Surg Pathol 2005;29:1625-1632.
12 Barthelmes L, Al Awa A, Crawford DJ. Effect of cavity margin shavings to ensure completeness of excision on local recurrence rates following breast conserving surgery. Eur J Surg Oncol 2003;29:644-648.

13 Malik HZ, Wilkinson L, George WD, et al. Preoperative mammographic features predict clinicopathological risk factors for the development of local recurrence in breast cancer. Breast 2000;9:329-333.

14 Andea AA, Wallis T, Newman LA, et al. Pathologic analysis of tumor size and lymph node status in multifocal/multicentric breast carcinoma. Cancer 2002;94:1383-1390.

15 Huston TL, Pigalarga R, Osborne MP, et al. The influence of additional surgical margins on the total specimen volume excised and the preoperative rate after breast-conserving surgery. Am J Surg 2006;192:509-512.

16 Rubin P, O’Hanlon D, Browell D, et al. Tumour bed biopsy detects the presence of multifocal disease in patients undergoing breast conservation therapy for primary breast carcinoma. Eur J Surg Oncol 1996;22:23-26.

17 Scopa CD, Aroukatos P, Tsamandas AC, et al. Evaluation of margin status in lumpectomy specimens and residual breast carcinoma. Breast J 2006;12:150-153.

18 Aziz D, Rawlinson E, Narod SA, et al. The role of reexcision for positive margins in optimizing local disease control after breast-conserving surgery for cancer. Breast J 2006;12:331-337.

19 Balch GC, Mithani SK, Simpson JF, et al. Accuracy of intraoperative gross examination of surgical margin status in women undergoing partial mastectomy for breast malignancy. Am Surg 2005;71:22-27.

20 Tartter PI, Kaplan J, Bleiweiss I, et al. Lumpectomy margins, reexcision, and local recurrence of breast cancer. Am J Surg 2000;179:81-85.

21 Luu HH, Otis CN, Reed Jr WP, et al. The unsatisfactory margin in breast cancer surgery. Am J Surg 1999;178:362-366. 